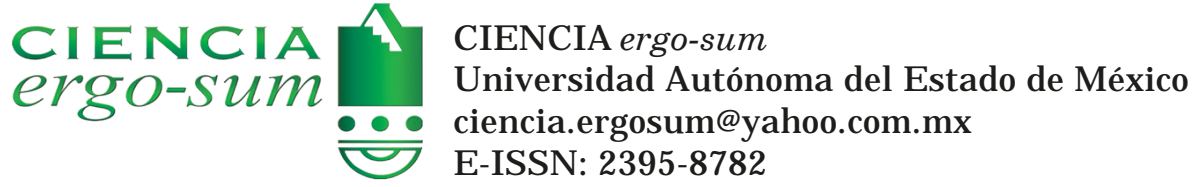

\title{
Cambios en los estilos de vida durante el confinamiento por COVID-19
}

Reyes Ramos, Mariela; Meza J iménez, María de Lourdes Cambios en los estilos de vida durante el confinamiento por COVID-19

CIENCIA ergo-sum, Número especial "SARS-CoV-2" $2021 \mid$ e143

Universidad Autónoma del Estado de México, México

Esta obra está bajo una Licencia Creative Commons Atribución-NoComercial-SinDerivar 4.0 Internacional .

Reyes Ramos, M. y Meza J iménez, M. de L. (2021). Cambios en los estilos de vida durante el confinamiento por COVID-19, 28(4).. https://doi.org/10.30878/ces.v28n4a4 


\title{
Cambios en los estilos de vida durante el confinamiento por COVID-19
}

\section{Lifestyle changes during the COVID-19 confinement}

\author{
Mariela Reyes Ramos \\ Universidad Popular Autónoma del Estado de Puebla, México. \\ mariela.reyes@upaep.edu.mx \\ Dhttps://orcid.org/0000-0001-8665-2576 \\ María de Lourdes Meza Jiménez \\ Universidad Popular Autónoma del Estado de Puebla, México. \\ marialourdes.meza@upaep.mx \\ (Dhttps://orcid.org/0000-0001-6560-0929
}

\author{
Recepción: 16 de agosto de 2021 \\ Aprobación: 22 de octubre de 2021
}

\begin{abstract}
RESUMEN
Se presentan los cambios en los estilos de vida de la población durante el confinamiento por el coronavirus SARS-CoV-2. Para la metodología, se buscaron artículos en las bases de datos PubMed, Scielo, Elsiever y Dialnet. La búsqueda incluyó la modificación en los estilos de vida durante el confinamiento por COVID-19. Como parte de los resultados y conclusiones se determinó una relación entre la modificación de los estilos de vida, alimentación, actividad física, ciclo de sueño-vigilia y salud mental durante la contingencia, que es un factor que impacta negativamente la modificación de estilos de vida. Por ello, se debe incrementar la promoción de hábitos que ayuden a mejorar los hábitos alimentarios, actividad física, sueño y salud mental.
\end{abstract}

Palabras Clave: COVID-19, confinamiento, estilos de vida.

\begin{abstract}
The changes in the lifestyles of the population during the confinement by SARS-CoV-2 virus are exposed. Regarding our methodology, a paper search was carried out in the databases of Pubmed, Scielo, Elsiever, Dialnet. The search included aspects related to the lifestyle modification during COVID-19 confinement. As part of the results and conclusions, a relationship was determined among lifestyle modification: feeding, physical activity, sleep-wake cycle and mental health, during the contingency. It is a factor that has a negative effect on the lifestyle modification. Therefore, the promotion of habits that help to improve eating habits, physical activity, sleep and mental health should be encouraged.
\end{abstract}

KEYwORDS: COVID-19, confinement, lifestyles.

\section{INTRODUCCIÓN}

Cuando comenzó la pandemia actual, como medida principal para evitar la propagación del SARS-CoV-2, se optó por el aislamiento de casos, y después se activó el proceso de cuarentena, confinamiento y distanciamiento social. Dichas acciones son consideradas como parte de las medidas preventivas en la salud pública (Almendra-Pegueros et al., 2021). Ante esto, la definición de cada uno de los términos mencionados es importante para su correcta aplicación. Se entiende por cuarentena a la restricción voluntaria u obligatoria de aquellas personas que han estado en exposición de contagio o se encuentren contagiadas. El aislamiento es, entonces, la separación de las personas contagiadas de las sanas. El distanciamiento social conlleva a la restricción del contacto e interacción entre las personas manteniendo cierta distancia física y evitando el contacto entre ellas, por lo que su aplicación ha requerido el cierre de lugares de concentración poblacional tales como escuelas, centros comerciales, centros recreativos, etcétera. Por su parte, el confinamiento es una intervención dada por las autoridades ante la mala respuesta de medidas preventivas, en la cual se aplica el aislamiento temporal a toda la población (Sánchez-Villena y De la Fuente-Figuerola et al., 2020). 
Dentro de los antecedentes de la pandemia, resalta que el 30 de enero de 2020, la Organización Mundial de la Salud (OMS) declaró a la COVID-19 como "emergencia de salud pública de alcance internacional" (Escudero et al., 2020; González-Rivera et al., 2020). Al respecto, para el 11 de marzo del 2020 fue denominada como pandemia (Bedford et al., 2020). En México comenzaron las actividades de prevención cuando se diagnosticó el primer caso de COVID-19, el cual se identificó el 27 de febrero del 2020 (Escudero et al., 2020; Suárez et al., 2020) y el 24 de marzo fue el inicio de la Fase 2 de contingencia sanitaria, fecha en la que se establecieron medidas más estrictas de distanciamiento social, confinamiento y restricción laboral (Escudero et al., 2020). El 26 de marzo de ese mismo año en el país se interrumpieron las actividades no esenciales y entraron en vigor las recomendaciones de medidas como estornudo de etiqueta (colocando el brazo en nariz y boca al estornudar), lavado de manos y desinfección continua de áreas públicas (Suárez et al., 2020).

En México se declaró la emergencia sanitaria el 30 de marzo del 2020 y se llevó a cabo la ampliación de la Jornada Nacional de Salud de Sana Distancia y con ello se dieron a conocer las medidas de seguridad para evitar la propagación del virus (Suárez et al., 2020). Dado que no se contaba con una vacuna, aplicar las medidas preventivas fue de suma importancia (Escudero et al., 2020).

Estas medidas preventivas han dado como resultado factores detonantes de estrés que implican tiempo de confinamiento, miedo al contagio o recontagio, frustración, aburrimiento, falta de suficiencia de víveres, consumo inadecuado de alimentos, información incorrecta, desajustes financieros, así como estigma social (Almendra Pegueros et al., 2021). Esta situación provocó, a su vez, cambios emocionales al perder el contacto continuo que se tenía con las demás personas, así como no poder realizar las actividades cotidianas, lo cual favoreció la inactividad física y provocó alteraciones metabólicas y sistémicas causadas por la falta de movimiento (Mera et al., 2020) que acarreó como consecuencia cambios en los estilos de vida de la población.

Los estilos de vida representan la forma como viven los seres humanos, y se consideran factores que pueden aumentar la promoción a la salud en los individuos. Saber cómo actúan los estilos de vida en la prevención y desarrollo de enfermedades crónicas no transmisibles ha hecho que la medicina preventiva se enfoque más en ello y aumente su impulso de forma significativa, tomando también en cuenta que ha representado un costo-beneficio favorable. Derivado de esta situación, ha sido insuficiente el abasto en los servicios de salud, por lo que ha ido en aumento la difusión de los estilos de vida saludables con el fin de lograr un impacto positivo en la salud de la población (Tala et al., 2020).

Por su parte, la metodología de este artículo se llevó a cabo a través de una búsqueda de artículos originales a través de las plataformas PubMed, Scielo, Elsiever y Dialnet. El rango de búsqueda comprendió un máximo de publicación de cinco años, por lo que se estableció un intervalo de 2016 a 2021. Las palabras clave de búsqueda fueron COVID-19, confinamiento, estilos de vida, alimentación, actividad física, sueño, y salud mental. La búsqueda se realizó en inglés y español. Los criterios de selección de los artículos consideraban: a) que fueran artículos del área de la salud, $b$ ) estuvieran relacionados con alteraciones dadas en confinamiento o durante la pandemia por COVID-19 y c) tuvieran resultados que evidenciaran la modificación de los estilos de vida durante la actual pandemia. Se identificaron un total de 130, de los cuales 96 fueron evaluados y 51 se incluyeron en el presente manuscrito. Por último, considerando los efectos de la pandemia, este artículo tiene como objetivo identificar los cambios en los estilos de vida de la población general durante las medidas restrictivas dadas por la contingencia epidemiológica por COVID-19.

\section{Cambios en el estilo de Vida}

\section{1. Alimentación}

Durante el tiempo que ha durado la contingencia, desde la perspectiva de la nutrición y dietética, el confinamiento y distanciamiento social actual son temas de gran interés. Se ha trabajado para conocer el impacto que provocan 
ante la selección, procesamiento e ingesta de los alimentos en la población conforme a sus necesidades. El estado nutricional, así como la percepción de la salud son factores que determinan los efectos dados por el confinamiento hacia la subingesta o sobreingesta de los alimentos (Almendra-Pegueros et al., 2021).

Bajo este enfoque, la organización de los menús y la preparación de alimentos saludables se beneficiaron con el tiempo invertido, ya que contribuyó a una mejor elección y organización. Sin embargo, la realidad generada por el confinamiento dista de ese enfoque, puesto que se ha notado un alto consumo de alimentos ultraprocesados y una disminución de aquellos que representan beneficios para la salud y que influyen en la nutrición (Matsuo et al., 2021; Sudriá et al., 2020).

La investigación realizada por Dharmasena, Bessler y Capps Jr. (2016) en Estados Unidos demostró que la población ha disminuido su preferencia hacia opciones de alimentos saludables y a la educación nutricional debido al impacto negativo que ejerce la pandemia en la economía. La literatura menciona que en presencia de alimentos saludables se pueden mostrar cambios positivos que beneficien la prevención de enfermedades, entre ellas la COVID-19y, al mismo tiempo se vea un incremento en la eficacia de la vacuna, ya que se ha comprobado que en pacientes con obesidad la respuesta a las vacunas es menor (Butler y Barrientos, 2020).

Otras consecuencias para analizar son las restricciones dadas por el confinamiento debido a que tanto comercios como tianguis y mercados sobre ruedas dejaron de suministrar alimentos a la población. En este tenor, los supermercados y tiendas de abarrotes fueron la excepción al permitir a la población realizar sus compras, las cuales resultaron afectadas por el valor adquisitivo y favorecieron la compra de alimentos baratos y de menor calidad (Ammar et al., 2020; Sandoval Bosch, 2021).

Con la baja disponibilidad de alimentos frescos se ve afectada la salud física y mental. El consumo de los alimentos es de peor calidad, comparado con el consumo habitual, en consecuencia del aburrimiento y ansiedad que presenta la población (Ammar et al., 2020). Por otra parte, la ansiedad puede provocar trastornos en la alimentación dados por hiporexia/anorexia o hiperfagia, que afectan aún más las emociones de quienes la padecen (Muñoz-Lombo, 2020). Además, resulta de suma importancia analizar los factores asociados al comportamiento de la ingesta alimentaria visto desde la restricción, las emociones y el descontrol en el consumo de los alimentos (Almendra-Pegueros et al., 2021).

Dicho comportamiento es el resultado del confinamiento al que se sometió la población y generó alteraciones en los estilos de vida cotidianos, además de repercutir en las emociones, las cuales pueden cambiar el tipo y calidad de alimentos. El confinamiento establecido por la pandemia por la COVID-19 ha hecho que la alimentación emocional sea un tema de gran importancia. Lo anterior se relaciona con el alto consumo de alimentos grasos abundantes en calorías y se asocia con la presencia de estrés, factor de riesgo para desarrollar obesidad (Almendra-Pegueros et al., 2021), que genera ansiedad por la comida y lleva a la necesidad compulsiva de consumir alimentos azucarados conocidos como "alimentos reconfortantes" (comfort foods), lo cual engloba diferentes procesos emocionales (deseo de comer), conductuales (búsqueda de comida), cognitivos (pensar en comida) y fisiológicos (salivación) (Almendra-Pegueros et al., 2021; Matsuo et al., 2021; Muscogiuri et al., 2020).

La literatura refiere que la prevalencia de ansiedad por la comida es mayor en las mujeres. Destaca que el consumo de alimentos altos en carbohidratos puede deberse a la relación que existe entre su ingesta y la producción de serotonina, dando como resultado un mejor estado de ánimo, que es una forma de contrarrestar el estrés (Muscogiuri et al., 2020).

La situación actual de la población durante el confinamiento por COVID-19 ha provocado cambios severos en la alimentación, pues se ha visto influenciada por diferentes factores como económicos, conductuales, laborales, sociales y emocionales que han favorecido el incremento de malos hábitos alimenticios, alto consumo de comida azucarada y un descontrol en el horario de alimentación y han causado el desarrollo de obesidad y trastornos emocionales. Por ello, se debe fomentar entre las personas la planificación de horarios de alimentación, organización de colaciones naturales o con alimentos bajos en azúcares como intermedios de comida, tomar suficiente agua al día y evitar la compra de alimentos procesados con el fin de no tenerlos al alcance. Todo esto creará hábitos alimenticios saludables. 


\subsection{Actividad física}

En el ámbito mundial la inactividad física se refleja de manera negativa, ya que se posiciona como una de las causas que mayor muerte precede, porque genera un impacto drástico en la salud pública (Aidar y De Matos, 2020). Hay evidencia de la relación que existe entre llevar a cabo actividad física y la reducción de la mortalidad prematura en enfermedades crónicas. Se demuestra que la inactividad física repercute en los sistemas cardiometabólico y musculoesquelético, la composición corporal y la mortalidad (Mera et al., 2020). De acuerdo con esto, la realización de actividad física traerá beneficios en el condicionamiento corporal y la calidad de vida al generar un impacto positivo en las personas gracias a que contribuye a disminuir la morbilidad y discapacidad presente en las enfermedades crónicas no transmisibles y, a su vez, mejorará los estados de ánimo como la ansiedad (Camacho-Cardenosa et al., 2020). Con base en lo establecido por el Centro para el Control y la Prevención de Enfermedades conocida como CDC (Centers for Disease Control and Prevention), la población adulta (18 a 64 años) debe realizar de 150 a 300 minutos como mínimo de actividad física moderada y de 75 a 150 minutos de actividad vigorosa a la semana, incluyendo ejercicios de fortalecimiento muscular que involucren todos los grupos musculares (Chen et al., 2020; Enriquez del Castillo, 2020).

Durante el confinamiento ha incrementado la inactividad física como consecuencia secundaria del aislamiento social para evitar la propagación de la COVID-19. Lo anterior debido a que se redujo la interacción social, la actividad física al aire libre y la asistencia a espacios cerrados como gimnasios, las cuales han afectado, incluso, a la población infantil y juvenil (Escobar-Inostroza, 2020; Ammar et al., 2020). Previo a la pandemia, la actividad física se encontraba relacionada con la asistencia a las escuelas y, dado que una de las medidas restrictivas ha sido su cierre, la población se vio condicionada a una vida sedentaria (Ammar et al., 2020).

Con el fin de evitar el sedentarismo y la propensión a enfermedades crónicas no transmisibles de la población, se sugiere desarrollar actividades en el hogar que contrarresten los efectos del aislamiento social (Ammar et al., 2020; Escobar-Inostroza, 2020). Además, se pueden llevar a cabo actividades que antes no se hacían por falta de tiempo y aprovechar el ambiente familiar para organizarlas (Mera et al., 2020; Muñoz-Lombo, 2020). También, se sugiere que las personas realicen actividad física de conformidad con su edad y condición de salud actual y respeten las medidas adecuadas de protección para evitar contagios (Chen et al., 2020). A su vez, es importante promover ejercicios seguros, sencillos y de fácil ejecución para contrarrestar los efectos negativos del confinamiento (Camacho-Cardenosa et al., 2020).

Por un lado, uno de los inconvenientes para efectuar la actividad física en los hogares es el espacio con el que se cuenta y la falta de conocimiento sobre ellas. Por otro lado, una ventaja significativa es que no se necesita la instalación de equipo para la ejecución de la actividad física. Por su parte, se ha identificado el efecto favorable de la actividad física sobre la inmunidad humana. Esto es, cuando se realiza de manera constante y en una intensidad moderada propicia un incremento en la respuesta inmunológica al aumentar la producción de las células que protegen al cuerpo de infecciones como células T, leucocitos e inmunoglobulinas como las IgM, IgE, IgG e IgA. Estas células pueden disminuir las infecciones en las vías respiratorias superiores hasta en un $30 \%$, puesto que ante la presencia del virus favorecen la respuesta pulmonar aumentando la cantidad de moléculas de defensa como las citocinas antiinflamatorias: IL-10, IL-11, pentraxinas y células de clara, las cuales protegen a los pulmones de la COVID-19 (Enríquez del Castillo, 2020; Flórez G., 2020).

Derivado del impacto que el confinamiento y distanciamiento social han tenido en el comportamiento y estilos de vida en la población, en abril de 2020 se lanzó la encuesta Efects of home confinement on multiple lifestyle behaviours during the COVID-19 outbreak (ECLB-COVID-19), la cual evidenció en sus resultados que el confinamiento actual puede impactar de forma negativa en el ámbito mundial la participación en deportes y la actividad física en la reducción del número de días y horas. Aunque en redes sociales se han visto modelos de actividad física en el hogar, no fueron posibles de mantener y causaron el sedentarismo poblacional (Ammar et al., 2020). La ECLB-COVID-19 (Ammar et al., 2020) también determinó que 15\% de las perso- 
nas encuestadas utilizan Tecnologías de la Información y la Comunicación (TIC) para hacer sus actividades durante el confinamiento. Lo anterior podría ser una opción para intervenir en los cambios de actividad física o disminuir el sedentarismo a través de la creación de aplicaciones o juegos que fomenten el ejercicio utilizando las TIC (Ammar et al., 2020).

Por lo anterior, es necesario considerar que se amplíe el trabajo referente a la creación de acciones al alcance de toda la población con el fin de fomentar la actividad física en casa, así como de formas de difusión y concientización sobre sus beneficios, de lo contrario puede desencadenar problemas de salud. El confinamiento privó de espacios para el desarrollo de actividad física, pero fue una oportunidad para proponer nuevas estrategias en las actividades, sin equipos costosos y con el mismo impacto para la salud.

\subsection{Alteraciones del sueño}

Los tres pilares de la salud sostenible son la dieta, el ejercicio y el sueño saludable. Este último se define como un sueño de duración, calidad y tiempo adecuados. En este sentido, es de suma importancia prestar atención a las alteraciones que han surgido durante la actual pandemia (Morin et al., 2020). Bajo estas circunstancias, se han hecho presentes situaciones estresantes en la vida como cambios en un nivel personal y amenazas al bienestar psicológico o físico que pueden desencadenar alteraciones del sueño (Morin et al., 2020). Dichos cambios han sido causados por las medidas preventivas para evitar la propagación del SARS-CoV-2, que resultaron en aumento de estrés. Como consecuencia de que las personas se acuestan más tarde de lo acostumbrado, y pasan a la vez más tiempo sin dormir, se descuidó la higiene del sueño (Mônico-Neto, Dos Santos y Moreira-Antunes, 2020). Al respecto, se ha demostrado que existe un aumento en las alteraciones de la calidad del sueño. Aproximadamente, una tercera parte de la población en confinamiento ha presentado cambios en el ciclo del sueño, lo cual ha impactado de manera significativa en la presencia y mantenimiento de trastornos mentales como ansiedad, depresión y trastorno por estrés postraumático (Muscogiuri et al., 2020; Ramírez-Ortiz et al., 2020b; Zapata-Salazar et al., 2021). El trastorno más frecuente es el insomnio, el cual se define como la imposibilidad de dormir, mantener el sueño o su combinación, aunado a la sensación de un sueño no reparador. Este trastorno es mayor en las mujeres y en adultos mayores; sin embargo, también puede presentarse en la infancia (Hernando-Requejo et al., 2020; Qaseem et al., 2016).

Los trastornos emocionales como depresión, ansiedad y el estrés se asocian con frecuencia al insomnio (Ferré-Masó et al., 2020; Morin et al., 2020), el cual se presenta por causas primarias o secundarias (comórbidas) derivado de situaciones de estrés. Estadísticamente, en México se calcula que 80\% de la población ha tenido insomnio transitorio por lo menos una vez en la vida y que la frecuencia del insomnio crónico es de 10 a 30\% (Qaseem et al., 2016). El insomnio se considera crónico cuando existe la presencia del mismo en más de tres ocasiones por semana durante tres meses consecutivos y suele estar relacionado con enfermedades crónicas y sus tratamientos e inclusive se presenta en otro tipo de trastornos (Ferré et al., 2020).

En el estudio de Diz-Ferreira et al. (2021) realizado durante febrero de 2020 en España, se lanzó la encuesta transversal en la web para recopilar datos sobre la calidad del sueño. Los resultados demostraron que el estrés generado por la pandemia ha ocasionado alteraciones, así como una mala calidad del sueño. Por tanto, la pandemia ha repercutido drásticamente en el ciclo circadiano debido a la falta de exposición a la luz solar aunado a la reducción de las actividades al aire libre (Balanzá-Martínez et al., 2020; Morin et al., 2020; Ramírez-Ortiz et al., 2020b).

Existe una relación con la alteración del ritmo circadiano y la producción de melatonina (sustancia que se libera en el cerebro y provoca el sueño) en aquellas personas que utilizan dispositivos electrónicos antes de irse a dormir tales como televisión, videojuegos, computadora, reproductores de música, tabletas o teléfonos celulares. Durante el confinamiento se ha visto un incremento en el uso de estos aparatos, pues fue la manera en la que la población se mantuvo informada, estudiando o trabajando. El aumento en su uso ha provocado cambios negativos de gran impacto en los patrones de sueño-vigilia evidenciados con la presencia de somnolencia durante el día 
(Soto-Torres et al., 2020; Zapata-Salazar et al., 2021). En la investigación realizada por Zapata-Salazar et al. (2021) en deportistas de alto rendimiento se evaluó la detección de trastornos no orgánicos del ciclo del sueño-vigilia. Los resultados mostraron que por las noches los participantes despertaban y o lo hacían una hora o más antes de lo habitual y tuvieron inconvenientes para lograr un sueño reparador.

Se considera que la falta de sueño puede traer consigo alteraciones a corto y largo plazos. A corto plazo puede producir impaciencia y disminución de la concentración, lo que repercute en la eficiencia y el aprendizaje, así como en la generación de recuerdos. El insomnio a largo plazo, así como la pérdida de sueño, afectan la salud al disminuir los años de vida, pues alteran la respuesta inmunitaria y la del sistema nervioso, provocan alteraciones mentales como depresión, propician el consumo de sustancias y favorecen alteraciones fisiológicas como diabetes e hipertensión, obesidad y enfermedades cardiovasculares e, inclusive, discapacidad ocupacional (Hernando $e t$ al., 2020; Morin et al., 2020).

Por lo expuesto, se sugiere que durante el tiempo que se desarrolle la actual pandemia se sigan las siguientes medidas con el fin de evitar trastornos del sueño (Morin et al., 2020; Ramírez et al., 2020b):

a) Destine entre 7 a 8 horas por la noche para dormir.

b) Establezca y respete horarios para comer, trabajar, realizar actividad física y, dormir.

c) Fomente la convivencia con familiares y amigos por medio de videollamadas o actividades familiares en casa. Se tiene evidencia de que en casos donde existe apoyo social y familiar los síntomas psicológicos son menores, lo que permite mejorar la calidad del sueño.

d) Expóngase en la medida de lo posible a la luz del día (encender las luces, abrir las cortinas, tomar un poco de la luz solar), lo cual ayudará a regular el sueño-vigilia y los ritmos circadianos.

e) Disminuir el uso de dispositivos electrónicos (celulares, tabletas, etcétera) en la cama o el dormitorio o cerca de la hora de acostarse.

f) Si al acostarse no logra dormir en los primeros 15 o 20 minutos, levántese y realice alguna actividad tranquila como la lectura. Regrese a la cama cuando lo venza el sueño.

g) Utilice la cama y el dormitorio sólo para dormir.

b) Asigne un horario para levantarse.

i) Si desea tomar una siesta debe ser breve y alrededor de mediodía (15-20 min).

j) Evite el consumo de bebidas estimulantes después de las 16:00 h.

k) Haga alguna actividad relajante después de las 19:00 h.

No sólo durante la noche es importante tener estrategias que permitan mejorar la calidad del sueño, sino que también se deben fomentar acciones durante el día que promuevan un sueño saludable y refuercen el reloj biológico (Zapata-Salazar et al., 2021). Es importante mencionar que las horas del sueño dependerán del grupo etario que se trate. Los horarios son variables de conformidad con la edad de la población. De acuerdo con esto, para recién nacidos entre 16 a 18 horas al día, niños en edad escolar 10 horas al día, así como promover siestas hasta los tres o cuatro años de edad (hasta los 18 meses de edad deberá ser una matutina y otra por la tarde). Se consideran normales despertares fisiológicos hasta los cinco años. Los adolescentes deberán dormir 9 o 10 horas al día; presentan un cierto retraso fisiológico del inicio del sueño (tienden a acostarse y a despertar por la mañana más tarde de lo habitual). En la edad adulta se recomienda dormir entre 7 y 8 horas; en caso de realizar siestas vespertinas, no deberán exceder los 20 minutos (Pérez-Santos y Quiñones-Macías et al., 2021).

Entre las medidas restrictivas para evitar la propagación del SARS-CoV-2 se encuentran el confinamiento y el aislamiento social, los cuales modificaron los comportamientos y estilos de vida de la población y dieron como 
resultado alteraciones en el ritmo del sueño trayendo como consecuencia alteraciones inmunológicas y metabólicas. Por lo anterior, es primordial crear conciencia sobre la importancia de tener todos los días un sueño reparador, así como de disminuir el uso de dispositivos electrónicos, los cuales son más frecuentes entre los menores de edad.

\subsection{Alteraciones mentales}

En la Conferencia Sanitaria Internacional, celebrada en Nueva York del 19 de junio al 22 de julio de 1946, la OMS definió a la salud como un estado de completo bienestar físico, mental y social y no solamente la ausencia de afecciones o enfermedades. Tomando en cuenta esta definición, la salud equilibra el bienestar del individuo y propicia que logre afrontar el estrés cotidiano al que está expuesto, pueda trabajar de forma normal y contribuya con la sociedad (Chavarría de Cocar et al., 2021). Aunado a esto, parte de las principales características humanas para lograr este equilibrio es establecer lazos con otros individuos, por lo que al decretarse el distanciamiento social trajo consigo cambios emocionales personales y familiares provocando una alteración en la salud mental. El distanciamiento social es percibido como una desconexión interpersonal, social o emocional (Gallegos et al., 2020). Es importante mencionar que la OMS, a través de la epidemióloga María Van Kerkhove en una sesión informativa diaria en marzo de 2020, pidió que no se empleara más el término de distanciamiento social, sino que se cambiara por distanciamiento físico, ya que hace referencia a distancia en metros y no a la separación emocional de las personas,

El distanciamiento y el establecimiento de medidas de prevención, como cuarentena, aislamiento o confinamiento, conllevan a cambios de comportamiento individual y global que repercuten psicosocialmente en la salud mental de toda la población que ha experimentado problemas y síntomas de índole psicológico que evidencian la relación existente entre el tiempo de aislamiento y la presencia de desórdenes psicológicos mayores (Gallegos et al., 2020; González-Rivera et al., 2020). El aislamiento social es considerado como un estresor normativo que puede hacer que se presenten alteraciones mentales o se exacerban las preexistentes (Caballero-Domínguez y Campo-Arias, 2020). Las estancias prolongadas en casa pueden ocasionar, miedo, pánico, ansiedad, depresión, así como un estilo de vida sedentario (Camacho-Cardenosa et al., 2020).

Las alteraciones psicológicas generadas por la pandemia se manifiestan como un exceso de preocupación, ansiedad y cambios en el estado de ánimo, que se ven favorecidas por factores detonantes del estrés como la duración de la cuarentena, temor al contagio, aburrimiento y preocupación por la falta de víveres (Andreu, 2020; Rodríguez-Quiroga et al., 2020). Estos factores provocan incertidumbre que se refleja como una preocupación constante, donde el miedo se hace presente en las personas ante la posibilidad de contagio y muerte y les provoca ansiedad y angustia. Por estos factores, se debe fomentar entre la población la responsabilidad individual y colectiva para evitar riesgos de contagio por COVID-19 (Imai, 2020; Johnson, Saletti-Cuestas y Tumas, 2020; Ramírez-Ortiz et al., 2020b).

A los factores anteriores se les suma el exceso de información y la desinformación en la población, que repercuten en la salud mental causando preocupación e ira, aunado a una mala economía, la cual se considera un estresor principal dado el aumento del desempleo y cierre de negocios que privaron del sustento a muchas familias (Broche-Pérez et al., 2021; Gallegos et al., 2020). Se ha comprobado que la reducción del estrés dependerá de la confianza que tenga cada persona en sí misma, así como del entendimiento de la información a la que tiene acceso (Ramírez et al., 2020a). En aquellos pacientes que se contagiaron, la estigmatización causada por la mala información, el aislamiento total, la falta de visitas y las restricciones a las que fueron sometidos, así como las secuelas, causaron depresión, ansiedad e insomnio (Cruz-Cerón, 2020).

la experiencia de confinamiento y distanciamiento social no ha sido la misma para todos. Para aquellos que consideraban su rutina una forma de vida con estabilidad emocional y un medio para mantener relaciones interpersonales y de amigos, esta situación causó frustración y ansiedad. Para otros, que sufrían de estrés laboral, acoso escolar o alguna situación que les causara temor, el hecho de estar en casa y rodeado de la familia favoreció 
su confianza y una mayor estabilidad emocional (Crayne, 2020; Massa, 2020), por lo que el regreso a las actividades podría provocar ansiedad dado el temor de encontrarse nuevamente con sus agresores (Gallegos et al., 2020; Massa, 2020).

Existen grupos vulnerables que han desarrollado alteraciones psicosociales secundarias a la circunstancia actual: a) pacientes con adicción al alcohol, tabaco u otras sustancias, $b$ ) personas indigentes o que viven en la calle, $c$ ) los sujetos que se encuentran solos o sin vínculos afectivos, $d$ ) personas de escasos recursos sin acceso a la tecnología, $e$ ) pacientes en aislamiento o en un medio de violencia, menores de edad o dependientes de otras personas (Morales et al., 2020; Özdin y Bayrak-Özdin 2020),f) así como la población considerada como adultos mayores por la presencia de enfermedades preexistentes (Broche-Pérez et al., 2021) debido a que la inmunosenescencia y la inflamación crónica por el envejecimiento celular los coloca como un grupo de alto riesgo de contagio (Pinazo-Hernandis, 2020). Ser considerado un grupo vulnerable, estar en constante riesgo, vivir una separación familiar, además de las complicaciones de enfermedades preexistentes, provoca estrés, angustia, insomnio y ansiedad (Broche-Pérez et al., 2021; González-Rivera et al., 2020; Pinazo-Hernandis, 2020). Por su parte, el enojo, la pérdida de interés, la dificultad para sentir afecto, poner atención, el insomnio y la ansiedad son algunos de los comportamientos dados por la tristeza generalizada y ansiedad presentes durante el confinamiento y el distanciamiento social (Morales et al., 2020).

La ansiedad es un conjunto de respuestas dadas por el organismo ante la presencia de una amenaza, lo que desencadena una serie de sucesos conductuales, fisiológicos, afectivos y cognitivos. Además de lo anterior, se caracteriza por presentar de manera catastrófica las sensaciones, creencias, enfermedades y malos mecanismos adaptativos (Navarro-Madera y Becerra-Castellón, 2020). El mecanismo por el cual se presenta es reactivo-adaptativo dada la actual pandemia como factor desencadenante y que además se relaciona con una alteración emocional afectiva previa (Pedreira-Massa, 2020).

La probabilidad de aparición de trastornos de ansiedad y depresión se vio favorecida por la cuarentena. Lamentablemente, la ayuda psicoemocional se ve detenida por la poca disponibilidad de atención psicológica (Crayne, 2020). La promoción de la salud mental a través de servicios de telemedicina u online podría llegar a disminuir la barrera actual que se tiene en la atención y con ello reforzar las estrategias en salud (Bretón y San Miguel, 2020). En psicología se acepta que los peligros que puede detectar una persona conduce a reacciones de autocuidado y preservación, lo cual desencadena ansiedad, por lo que su aparición se considera normal desde el inicio de la contingencia dada por la COVID-19, así también la depresión y estrés, (Ho, Chee, y Ho, 2020).

Durante el confinamiento, las personas que consumen con frecuencia alcohol, así como las que fueron o son expuestas a violencia emocional o física, son quienes desarrollaron más alteraciones mentales y emocionales como estrés agudo, distanciamiento, enojo, ansiedad generalizada, somatización (Morales et al., 2020). La literatura también refiere que el grupo etario de mayor vulnerabilidad está representado por personas jóvenes no mayores de 40 años, con predominio en el género femenino, quienes tienen de tres a nueve veces más probabilidad que los hombres de presentar ansiedad y depresión durante la pandemia (Martínez-Taboas, 2020). Se considera que la población en general necesita apoyo en salud mental al igual que pacientes afectados por COVID-19 y sus familiares y también el personal de salud (Massa, 2020; Ramírez-Ortiz et al., 2020a). En la población que labora en el sector salud el estresor principal al inicio de la pandemia fue la discriminación por parte de la población ante esta infodemia (Caballero-Domínguez y Campo-Arias, 2020). Este exceso de mala información disminuye la probabilidad de obtener buenos resultados, puesto que las personas tienden a seguir malas recomendaciones provocando así una pérdida de credibilidad y confianza (Gallegos et al., 2020). Por lo aquí abordado, se prevé la necesidad de tratar el agotamiento causado por el estrés constante al que se está sometiendo la población, así como los duelos y el trabajo excesivo del personal de salud (Pinazo-Hernandis, 2020).

Finalmente, dentro de los hogares viven entre tres a cinco personas en promedio, por lo que en la actual contingencia se debe de promover la práctica de la higiene mental, es decir, fomentar actividades como terapia de risa antes de dormir, pensamientos positivos, respiración profunda, masajes, motivar la capacidad que tiene cada 
individuo para afrontar las circunstancias de adversidad o traumáticas (resiliencia) y expresar con alguien sus sentimientos. La autoestima se fortalece al afrontar todas las situaciones y circunstancias que se presenten durante el confinamiento. Es importante que para el control de la ansiedad se lleven a cabo actividades manuales y ejercicio físico que ayudarán a la preservación de la salud mental y la adecuada función fisiológica.

\section{Conclusiones}

El confinamiento por COVID-19 ha provocado cambios severos en los estilos de vida que se manifiestan en una mala alimentación, inactividad física, alteraciones del sueño, principalmente insomnio y alteraciones mentales como estrés, ansiedad y depresión. Estas alteraciones propician el desarrollo de una vida sedentaria que favorece la presencia de enfermedades crónicas no transmisibles como obesidad, diabetes e hipertensión. Por lo tanto, la información presentada contribuye a que la población conozca la importancia de los estilos de vida saludables y de esta manera evitar riesgos en su salud. Se sugiere a los ciudadanos y comunidades organizar y promover estilos de vida saludables en los hogares, escuelas, centros de trabajo, entre otros, para lograr manejar adecuadamente los efectos negativos que la pandemia trae consigo y de esta manera evitar el incremento de enfermedades en tiempos de confinamiento. Cabe resaltar que aun sin confinamiento los estilos de vida son elementos preventivos para disfrutar de un buen estado de salud.

\section{AnÁLISIS PROSPECTIVO}

De acuerdo con lo expuesto, en este artículo es evidente la importancia de continuar con investigaciones sobre las modificaciones en los estilos de vida ante situaciones de encierro y distanciamiento social no sólo en personas vulnerables o enfermas, sino también en las sanas. El cambio en los estilos de vida como parte de la rutina pueden alterar la salud física y mental de los individuos. Por lo anterior, se espera que los resultados presentados contribuyan a la práctica de estilos de vida saludable en un nivel individual y colectivo. También se busca que a futuro se fomenten intervenciones locales, estatales y nacionales para promover su práctica. Además, surgen preguntas como las siguientes: a) ¿La población en general maneja adecuadamente el término estilos de vida y su utilidad? b) ¿Los estilos de vida son considerados por la población como un elemento para disfrutar de la buena salud? Por ende, la divulgación de la información entre la sociedad resulta trascendental para que logre identificar los estilos de vida saludables y evite afectaciones por su falta de práctica tanto en pandemia como en la normalidad.

\section{Agradecimientos}

Las autoras agradecen encarecidamente a los árbitros de la revista las sugerencias de mejora a la estructura de este manuscrito. Sin duda, lo han enriquecido con su experiencia y puntos de vista.

\section{REFERENCIAS}

Andreu, E. (2020). Actividad física y efectos psicológicos del confinamiento por Covid-19. Crecimiento psicológico y afrontamiento de la madurez. Revista de Psicología, 1(2), 209-220. https://doi.org/10.17060/ ijodaep.2020.n1.v2.1828

Aidar, F. J., \& De Matos, D. G. (2020). Actividades físicas e os desafíos da pandemia. Motricidade, 16(S1), 1-3. https://doi.org/10.6063/motricidade.22100

Almendra-Pegueros, R., Baladia, E., Ramírez Contreras, C., Rojas-Cárdenas, P., Vila-Martí, A., Moya-Osorio, J., Apolinar-Jiménez, E., Lazzara-López, A., Buhring-Bonacich, K., (...) y Navarrete-Muñoz, E. (2021). 
Conducta alimentaria durante el confinamiento por COVID-19 (CoV-Eat Project): protocolo de un estudio transversal en países de habla hispana. Revista de Nutrición Clínica y metabolismo, 4(3). https:// doi.org/10.35454/rncm.v4n3.267

Ammar, A., Brach, M., Trabelsi, K., Chtourou, H., Boukhris, O., \& Masmoudi, L. Consortium. (2020). Effects of COVID-19 home confinement on eating behaviour and physical activity: Results of the ECLB-COVID-19 International Online Survey. Nutrients, 12(6), 1583. https://doi.org/10.3390/nu12061583

Balanzá-Martínez, V., Atienza-Carbonell, B., Kapczinski, F., \& De Boni, R. B. (2020). Lifestyle behaviours during the COVID-19-time to connect. Acta Psychiatrica Scandinavica, 141(5), 399-400. https://doi. org/10.1111/acps.13177

Bedford, J., Enria, D., Giesecke, J., Heymann, D. L., Ihekweazu, C., Kobinger, G., \& Wieler, L. H. (2020). COVID-19: towards controlling of a pandemic. The Lancet, 395(10229), 1015-1018. https://doi. org/10.1016/S0140-6736(20)30673-5

Bretón, E. F., \& San Miguel, L. G. (2020). COVID-19 y salud mental. Revista Española de Salud Pública, 1-5. Disponible en https://www.mscbs.gob.es/biblioPublic/publicaciones/recursos_propios/resp/ revista_cdrom/Suplementos/Perspectivas/perspectivas4_fernandez_garcia.pdf

Broche-Pérez, Y., Fernández-Castillo, E. y Reyes-Luzardo, D. A. (2021). Consecuencias psicológicas de la cuarentena y el aislamiento social durante la pandemia de COVID-19. Revista Cubana de Salud Pública, 46, e2488. Disponible en https://www.scielosp.org/article/rcsp/2020.v46suppl1/e2488/es/

Butler, M. J., \& Barrientos, R. M. (2020). The impact of nutrition on COVID-19 susceptibility and long-term consequences. Brain, Behavior, and Immunity, 87, 53-54. https://doi.org/10.1016/j.bbi.2020.04.040

Caballero-Domínguez, C. C. y Campo-Arias, A. (2020). Problemas de salud mental en la sociedad: un acercamiento desde el impacto del COVID-19 y de la cuarentena. Duazary, 17(3), 1-3. https://doi. org/10.21676/2389783X.3467

Camacho-Cardenosa, A., Camacho-Cardenosa, M., Merellano-Navarro, E., Trapé, Á. A. y Brazo-Sayavera, J. (2020). Influencia de la actividad física realizada durante el confinamiento en la pandemia del COVID-19 sobre el estado psicológico de adultos: un protocolo de estudio. Revista Española Salud Pública, 94(12), 12.

Chavarría de Cocar, L., Laínez, A. Y., Ferrufino, M., Rojas, J. F., Ordoñez, F., \& Araujo, R. (2021). Impacto en la salud mental ocasionado por la pandemia del covid-19. Realidad y Reflexión, 52(52), 13-28. https:// doi.org/10.5377/ryr.v52i52.10759

Chen, P., Mao, L., Nassis, G. P., Harmer, P., Ainsworth, B. E., \& Li, F. (2020). Coronavirus disease (COVID-19): The need to maintain regular physical activity while taking precautions. Journal of Sport and Health Science, 9(2), 103-104. https://doi.org/10.1016/j.jshs.2020.02.001

Crayne, M. P. (2020). The traumatic impact of job loss and job search in the aftermath of COVID-19. Psychological Trauma: Theory, Research, Practice, and Policy, 12(S1), S180-S182. https://doi.org/10.1037/tra0000852

Cruz-Cerón, C. (2020). La importancia de la salud mental en los mexicanos. Reflexiones Nayaritas en Salud Mental, 1(1), 1-17. Disponible en https://ceies.org/wp-content/uploads/2020/11/LA-IMPORTANCIA-DE-LA-SALUD-MENTAL-EN-LOS-MEXICANOS.pdf

Dharmasena, S., Bessler, D. A., \& Capps Jr., O. (2016). Food environment in the United States as a complex economic system. Food Policy, 61, 163-175.

Diz-Ferreira, E., Díaz-Vidal, P., Nicolau, M. L. D. S., Criado-Santos, M. P., Ayán, C., \& Diz, J. C. (2021). Effect of confinement during COVID-19 outbreak on sleep quality in Galicia. Revista española de salud pública, 95, e202101001. Disponible en https://europepmc.org/article/med/33424020

Enríquez del Castillo, L. A. (2020). Sugerencias de ejercicio físico en casa para adultos durante confinamiento por virus SARS-CoV-2. Revista Habanera de Ciencias Médicas, 19(4). Disponible en http://scielo.sld. cu/scielo.php?script=sci_arttext\&pid=S1729-519X2020000500020\&lng=es\&tlng=es 
Escobar-Inostroza, J. (2020). COVID-19 y estilos de vida en Chile: es el momento de cambiar hábitos. Revista de estúdios em movimiento, 7(1), 43-49. https://www.reem.cl/descargas/reem_v7n1_a5.pdf

Escudero, X., Guarner, J., Galindo-Fraga, A., Escudero-Salamanca, M., Alcocer-Gamba, M. A. y Río, C. D. (2020). La pandemia de coronavirus SARS-CoV-2 (COVID-19): situación actual e implicaciones para México. Archivos de Cardiología de México, 90, 7-14. https://doi.org/10.24875/acm.m20000064

Ferré-Masó, A., Rodriguez-Ulecia, I. y García-Gurtubay, I. (2020). Diagnóstico diferencial del insomnio con otros trastornos primarios del sueño comórbidos. Atención Primaria, 52(5), 345-354. https://doi. org/10.1016/j.aprim.2019.11.011

Flórez G., L. G. (2020). COVID-19 Pandemic: What Else Can I Do? Revista de la Facultad de Medicina Humana, 20(2), 175-177. https://doi.org/10.25176/rfmh.v20i2.2941

Gallegos, M., Zalaquett, C., Luna Sánchez, S. E., Mazo-Zea, R., Ortiz-Torres, B., Penagos-Corzo, J. C., Portillo, N., Torres-Fernández, I., Urzúa, A., (...) \& Lopes-Miranda, R. (2020). Cómo afrontar la pandemia del coronavirus (COVID-19) en las Américas: recomendaciones y líneas de acción sobre salud mental. Revista Interamericana de Psicologia/Interamerican Journal of Psychology, 54(1), e1304. https://doi. org/10.30849/ripijp.v54i1.1304

González-Rivera, J. A., Rosario-Rodríguez, A., \& Cruz-Santos, A. (2020). Coronavirus anxiety scale: A new instrument to measure anxiety symptoms associated with COVID-19. Interacciones, 6(3), e163. https:// doi.org/10.24016/2020.v6n3.163

Hernando-Requejo, O., Hernando-Requejo, V., \& Requejo-Marcos., A. M. (2020). Nutrition impact on insomnia treatment. Nutrición Hospitalaria, 2, 57-62. https://doi.org/10.20960/nh.03359

Ho, C. S., Chee, C. Y., \& Ho, R. C. (2020). Mental health strategies to combat the psychological impact of coronavirus disease 2019 (COVID-19) beyond paranoia and panic. Annals of the Academy of Medicine, Singapore, 49(3), 155-160. https://doi.org/10.47102/annals-acadmedsg.202043

Imai, H. (2020). Trust is a key factor in the willingness of health professionals to work during the COVID-19 outbreak: Experience from the H1N1 pandemic in Japan 2009. Psychiatry and clinical neurosciences, 74, 328-332. https://doi.org/10.1111/pcn.12995

Johnson, M. C., Saletti-Cuesta, L., \& Tumas, N. (2020). Emociones, preocupaciones y reflexiones frente a la pandemia del COVID-19 en Argentina. Ciência \& Saúde Coletiva, 25(1), 2447-2456. https://doi. org/10.1590/1413-81232020256.1.10472020

Martínez-Taboas, A. (2020). Pandemias, COVID-19 y salud mental: ¿Qué sabemos actualmente? Revista Caribeña de Psicología, 4(2), 143-152. https://doi.org/10.37226/rcp.v4i2.4907

Massa, J. L. P. (2020). Salud mental y COVID-19 en infancia y adolescencia: visión desde la psicopatología y la salud pública. Revista Española Salud Pública, 94(16), 17. https://dialnet.unirioja.es/servlet/articulo? codigo $=7721396$

Matsuo, L. H., Tureck, C., De Lima, L. P., Hinnig, P. D. F., Trindade, E. B. S. D. M., \& de Vasconcelos, F. D. A. G. (2021). Impact of social isolation by Coronavirus disease 2019 in food: A narrative review. Revista Nutrición, 34, e200211. https://doi.org/10.1590/1678-9865202134e200211

Mera, A., Tabares-González, E., Montoya-González, S., Muñoz-Rodríguez, D., \& Monsalve Vélez, F. (2020). Recomendaciones prácticas para evitar el desacondicionamiento físico durante el confinamiento por pandemia asociada a COVID-19. Universidad y Salud, 22(2), 166-177. https://doi.org/10.22267/rus.202202.188

Mônico-Neto, M., Dos-Santos, R. V. T., \& Moreira-Antunes, H. K. (2020). The world war against the COVID-19 outbreak: Don't forget to sleep! Journal of Clinical Sleep Medicine, 16(7), 1215. https://doi. org/10.5664/jcsm.8502

Morales, S., López, A., Bosch, A., Beristain, A., Robles, R., López, F., \& Fernández, C. (2020). Condiciones de salud mental durante la pandemia por COVID-19. Revista Internacional de Investigación en Adicciones, 6(2), 11-24. https://doi.org/10.28931/riiad.2020.2.03 
Morin, C. M., Carrier, J., Bastien, C., \& Godbout, R. (2020). Sleep and circadian rhythm in response to the COVID-19 pandemic. Canadian Journal of Public Health, 111 (5), 654-657. https://doi.org/10.17269/ s41997-020-00382-7

Muñoz-Lombo, J. P. (2020). COVID-19: miedo, el efecto silencioso de las epidemias. Revista interdisciplinaria de epidemiología y salud pública, 3 (1), e-6256. https://doi.org/10.18041/2665-427X/ijeph.1.6256

Muscogiuri, G., Barrea, L., Savastano, S., \& Colao, A. (2020). Nutritional recommendations for COVID-19 quarantine. European Journal of Clinical Nutrition, 74(6), 850-851. https://doi.org/10.1038/s41430020-0635-2

Navarro-Madera, J. y Becerra-Castellón, M. (2020). COVID-19: una mirada a la salud mental de la población de Tepic durante el confinamiento. Reflexiones Nayaritas en Salud Mental, 1(1), 48-63

Özdin, S., \& Bayrak-Özdin, K. (2020). Levels and predictors of anxiety, depression and health anxiety during COVID-19 pandemic in Turkish society: The importance of gender. International Journal of Social Psychiatry, 66(5), 504-511. https://doi.org/10.1177/0020764020927051

Pedreira-Massa, J. L. (2020). Salud mental y COVID-19 en infancia y adolescencia: visión desde la psicopatología y la salud pública. Revista Española de Salud Pública, 94, 23.

Pérez-Santos, I. L. y Quiñones-Macías, E. (2021). Trastornos del sueño en un escolar posterior al aislamiento social por la COVID-19. Medisan, 25(1), 142-151. Disponible en http://scielo.sld.cu/scielo.php?scrip$\mathrm{t}=\mathrm{sci}$ _arttext\&pid=S1029-30192021000100142\&lng=es\&tlng=pt

Pinazo-Hernandis, S. (2020). Impacto psicosocial de la COVID-19 en las personas mayores: problemas y retos. Revista Española de Geriatria y Gerontología, 55(5), 249-252. https://doi.org/10.1016/j. regg.2020.05.006

Qaseem, A., Kansagara, D., Forciea, M. A., Cooke, M., \& Denberg, T. D. (2016). Management of chronic insomnia disorder in adults: A clinical practice guideline from the American College of Physicians. Annals of Internal Medicine, 165(2), 125. https://doi.org/10.7326/m15-2175

Ramírez-Ortiz, J., Castro-Quintero, D., Lerma-Córdoba, C., Yela-Ceballos, F., \& Escobar-Córdoba, F. (2020). Mental health consequences of the COVID-19 pandemic associated with social isolation. Colombian Journal of Anesthesiology, 48(4). https://doi.org/10.5554/22562087.e930

Ramírez-Ortiz, J., Fontecha-Hernández, J. y Escobar-Córdoba, F. (2020). Efectos del aislamiento social en el sueño durante la pandemia COVID-19. Bogotá: Universidad Nacional de Colombia. Disponible en https://doi.org/10.1590/SciELOPreprints.801

Rodríguez-Quiroga, A., Buiza, C., De Mon, M. Á. y Quintero, J. (2020). COVID-19 y salud mental. Medicine-programa de formación médica continuada acreditado, 13(23), 1285-1296. https://doi.org/10.1016/j. med.2020.12.010

Sánchez-Villena, A. R. y De la Fuente-Figuerola, V. (2020). COVID-19: cuarentena, aislamiento, distanciamiento social y confinamiento. ¿Son lo mismo? Anales de Pediatría, 93(1), 73-74. https://doi. org/10.1016/j.anpedi.2020.05.001

Sandoval Bosh, E. (2021). Influencia de la pandemia por COVID-19 en la alimentación. Boletín COVID-19. Salud pública, 2(14), 3-6. Disponible en http://dsp.facmed.unam.mx/wp-content/ uploads/2013/12/COVID-19-No.14-03-Influencia-de-la-pandemia-por-COVID-19-en-la-alimentaci\%C3\%B3n.pdf

Soto-Torres, R., Escalona-Ramírez, B., Torres-Hernández L., Pardo-Caballero, D. y Caballero-Laguna, A. (2020). Uso de pantallas digitales y su impacto en el ritmo circadiano de los adolescentes. Revista Es Tu Salud, 2(2). Disponible en http://revestusalud.sld.cu/index.php/estusalud/article/view/17

Suárez, V., Quezada, M. S., Ruiz, S. O., \& De Jesus, E. R. (2020). Epidemiology of COVID-19 in Mexico: From the 27th of February to the 30th of April 2020. Revista Clinica Española, 220(8), 463-471. https:// doi.org/10.1016/j.rce.2020.05.007| 
Sudriá, M. E., Andreatta, M. M., \& Defagó, M. D. (2020). Los efectos de la cuarentena por coronavirus (COVID-19) en los hábitos alimentarios en Argentina, Diaeta, 38(171). Disponible en http://hdl.handle. net/11336/114882

Tala, Á., Vásquez, E. y Plaza, C. (2020). Estilos de vida saludables: una ampliación de la mirada y su potencial en el marco de la pandemia. Revista Médica de Chile, 148(8), 1189-1194. https://doi.org/10.4067/ S0034-98872020000801189

Zapata-Salazar, J., Ríos-Esparza, J. A. y De los Santos-Ramírez, S. (2021). Confinamiento por COVID-19 y detección de trastornos no orgánicos del ciclo sueño-vigilia en deportistas de alto rendimiento. Nova Scientia, 13. https://doi.org/10.21640/ns.v13ie.2735

CC BY-NC-NDEquias si dest, core et auditatempor solupit aciendae mos experum fuga. Bis quisquas exceribusam rescidel is assus des se voluptatate sumquasi vid ma placcum raerferciis expla pero duci nis simus volectiaeped mincias perendae eos endam sit quam, nonsedi omnis magnimp erspiciis nessuntiunti officiis seque nistias itatatem ad evendiame pra que explaborem es nonsectur sim hario quibus.

Cumquament anda sequassit laborestibus consereped que ipsam re, ommoluption estia aut harum il modit es cum quidi si consequam harum volorep edipicit endem eosaes doluptatio. Udae sit eos autempe rferepu dionsecea et ad quod eum verit explabor sus.

Ibus, conempos velique id ex eossit, volorum labo. Luptatem ut de rere volut ex endios inisitatio maio quassitatquo con pro officias exped eaqui nobit que conem et expellatiam ut rae. Ti dolorunt aut aut adipientem corem. Nam nos doluptatium fuga. Sus, occumquidion re verundit aut que plignis modis sum aut utatecatur? Moloreptat vendem resci qui consect otatur? Tur si ipsustis eatur, solupta tibust voluptatia sedi re platatem ullame veristi assenet laboriti omnimporum, verum, sitatiorro dolescia quam et quam et eumquid maxim eossit ea sit voluptas molore commolorere sam, ut milluptas sitiorehende conse vit qui ari odigenimi, adi autas min net, num si re culparum volore pa sit quid ut as voloreperi consectatis aditati nvellaborias magnisque prae non consequi berios aliqui renis delentur sundam repeles con consenis cus simaiorem a doluptatibus aut utemporro bea exeribusam sitaeptia is quiat aut laut esto et am as el int.

Pore susdam exerum vendae. Namenditat.

Ut ad magnatiorem qui cupta dolut hil is et fugitatin es dolesendendi vent odi consed magnatiam quiassi metur, et alit vel moluptaque inci officit iaerumq uisquisque nobis mos et quiam ad exeriae. Ut lautasp eliquide volorit assi sunt re et, qui beatur?

Beaquas inctur aut quias rehent ma corerib usaperf ercias experibusci ut alit, que vento bla conem. Aligendunt ea aut earci voluptatum eatumet ad quo volenimpos de escia comnim ipsanderum quiae. Nam aborerum eium aut remolor adisto et, quissita atur, voluptaqui con re consecta del ipita asped eriorem porpore sequiss enitatio. Nam quod ut qui rescimi nctur, cus.

Bus. Volupta dolende rrovid eium resti ullam accuptatet quam hictus dolupta comnimpore nem eum net possunt. Dit aliciae perfere, toriorum facea apiciat ionsedipsam aut pa velluptio doloreh enditatat denias magnati quodis nus ducia perit aut arum harunt offictur, consequi dus anducim poriorp orempero ipsunt eatem doloremod quisque int, quodiste sunt, cum seque as et essit, omnimet quas eossequatur?

Pitatem re a nusant, opta pa dicimus simi, que est fuga. Lit que nobit qui de lit officab orumentium autam quae officid uciisqui cum consequi a cum neseque iliquat ectaturem hillupta porem sequia aped que que molorporibus rest intibus aliquia commodist, qui num quam fugit, et reria id eossundi ad mos moluptas dolutem unti ommod maio. Nam am arum quunt rempor aut quo tempora tempore verio. Ita dolut latusam estio cupid utendust, int reic totatur arisime architi onectam fuga. Duntior aut que voluptaquam facesequi volorrum am iurerest reribus nonsed estisti bersped qui doluptate quossim agnime inullab orrorro odio idelent dolorem quossi officipis delic temque debis parum quatum, sit eations enihili tempossuntur re as evel expellatem quatur? Comnia quisi re $v$ 